\title{
A reafferent model of song syntax generation in the Bengalese finch
}

\author{
Alexander Hanuschkin ${ }^{1,2^{*}}$, Markus Diesmann $n^{2,3,4}$, Abigail Morrison 1,2,3 \\ From Nineteenth Annual Computational Neuroscience Meeting: CNS*2010 \\ San Antonio, TX, USA. 24-30 July 2010
}

The Bengalese finch produces a set of ordered sequences of syllables. After deafening this song syntax is disrupted, i.e. within days the sequence become randomized and unstable [1]. Interestingly, the normal song syntax is recovered when hearing is restored [2]. Studies have shown that the vocal motor control system of the Bengalese finch rely on real time auditory feedback [3] and that activity in the high vocal center (HVC) is affected by feedback perturbations [4]. This suggests a reafferent model [3] of song syntax generation in which the perception of the bird's own song (BOS) cues the motor system.

Here, we present a functional network model of the song syntax generation based on realistic spiking neurons. Neurons are connected in feed-forward structures (synfire chains, SFCs) that can reproduce the neural activity observed in the HVC of the songbird [5]. Individual syllables are represented by the activity propagation throughout distinct SFCs. The auditory perception of the syllables is modeled by activity changes in an auditory network which in turn primes specific subsets of the HVC neurons to obtain the desired song syntax. If the auditory feedback is suppressed random syllable sequences are generated due to the 'winner takes all' competition of individual syllables [6].

\section{Conclusion}

Our model can reproduce the experimentally observed song syntax of the Bengalese finch and its disruption when auditory feedback is interrupted. It provides a framework for theoretical investigations of HVC activity and changes in the song syntax in response to specific feedback disturbances. Additionally, the model predicts priming of HVC neurons at the transition between individual syllables that could be tested in further experimental studies.

\footnotetext{
* Correspondence: hanuschkin@bccn.uni-freiburg.de

${ }^{1}$ Functional Neural Circuits, Faculty of Biology, Albert-Ludwig University of Freiburg, Germany
}

From a theoretical point of view the individual syllables can be regarded as primitives of the song which are combined following a given syntax. Hence, our reafferent model demonstrates how compositionality of a system can be realized given neurobiologically realistic assumptions.

\section{Acknowledgements}

Partially funded by DIP F1.2, BMBF Grant 01GQ0420 to BCCN Freiburg, EU Grant 15879 (FACETS), Helmholtz Alliance on Systems Biology (Germany), and Next-Generation Supercomputer Project of MEXT (Japan). All simulations are performed using NEST [7].

\section{Author details}

${ }^{1}$ Functional Neural Circuits, Faculty of Biology, Albert-Ludwig University of Freiburg, Germany. ${ }^{2}$ Bernstein Center Freiburg, Albert-Ludwig University of Freiburg, Germany. ${ }^{3}$ RIKEN Brain Science Institute, Wako City, Japan. ${ }^{4}$ RIKEN Computational Science Research Program, Wako City, Japan.

Published: 20 July 2010

\section{References}

1. Okanoya K, Yamaguchi A: Adult bengalese finches (lonchura striata var. domestica) require real-time auditory feedback to produce normal song syntax. Journal of Neurobiology 1997, 33(4):343-356.

2. Woolley SMN, Rubel E W: Vocal Memory and Learning in Adult Bengalese Finches with Regenerated Hair Cells. J. Neurosci. 2002, 22(17):7774-7787.

3. Sakata J T, Brainard M S: Real-Time Contributions of Auditory Feedback to Avian Vocal Motor Control. J. Neurosci. 2006, 26(38):9619-9628.

4. Sakata J T, Brainard MS: Online Contributions of Auditory Feedback to Neural Activity in Avian Song Control Circuitry. J. Neurosci. 2008, 28(44):11378-11390.

5. Hahnloser R H, Kozhevnikov A A, Fee M S: An ultra-sparse code underlies the generation of neural sequences in a songbird. Nature 2002, 419(6902):65-70.

6. Hanuschkin A, Herrmann J M, Morrison A, Diesmann M: A model of free monkey scribbling based on the propagation of cell assembly activity. BMC Neuroscience 2009, 10(Suppl 1):300.

7. Gewaltig M-O, Diesmann M: NEST (NEural Simulation Tool). Scholarpedia 2007, 2(4):1430.

doi:10.1186/1471-2202-11-S1-P33

Cite this article as: Hanuschkin et al:: A reafferent model of song syntax generation in the Bengalese finch. BMC Neuroscience 2010 11(Suppl 1): P33. 